\title{
Relaunching the African Pollen Database: Abrupt change in climate and ecosystems
}

\author{
Sarah Ivory', A.-M. Lézine², E. Grimm³ and J.W. Williams ${ }^{4}$
}

Paris, France, 13-16 October 2019

African ecosystems hold enormous ecological and economic value due to high biodiversity (Myers et al. 2000) and valuable ecosystem services provided to urban and agrarian populations (Wangai et al. 2016). However these services are vulnerable to land use and climate change (Niang et al. 2014). Long paleoecological records from Africa provide iconic examples of abrupt environmental change, offering critical evidence for tipping points in the Earth system. Datasets in the region are notoriously difficult to access with the African Pollen Database (APD) largely unsupported for the last decade. Poor data accessibility has been a community complaint.

Hosted by the Institut de Recherche pour le Développement in Paris, France, 32 participants from 12 countries met to revive efforts by the African paleoecology community and relaunch the APD as a community curated data resource (Williams et al. 2018). This workshop was organized by the LOCEAN and funded by the Belmont Forum for Science-driven e-Infrastructure Innovation project "Abrupt Change in Climate and Ecosystems: Where are the Tipping Points?" The Belmont e-infrastructure initiative seeks to enhance open, FAIR, scientific cyberinfrastructure (Wilkinson et al. 2016) to meet pressing, policy-relevant challenges.

\section{African Pollen Database: History and plan} The APD was first developed in 1996. The initial workshop and subsequent work, funded by the French CNRS, the EU (INCO, ENRICH) and UNESCO (PICG), established methods of collating pollen data, developed a standardized pollen nomenclature (Vincens et al. 2007), generated updated age models, collated images of pollen grains, and created a searchable web interface. Currently, the APD contains 288 fossil sites and 1985 modern samples. Due to a lack of funding and the closure of the French data center, MediasFrance, the APD lapsed in 2007, making its extant data holdings largely inaccessible and leaving no home for newer paleoecological records.

Workshop participants identified three critical elements of the APD: (1) Pollen samples with associated chronological controls and other metadata; (2) Standardized taxonomy for pollen morphotypes; and (3) Pollen grain images. Participants developed a two-pronged plan for rebuilding these APD components. The APD webpage will be relaunched, in collaboration with LSCE (IPSL, France) to meet region-specific needs, including modern and paleo data, taxonomy development, and hosting of pollen images. Further, APD data will become a constituent database of the Neotoma Paleoecology Database (Goring et al. 2015), an international community-curated data resource that meets FAIR standards and is registered with ICSU-WDS and COPDESS. Planning began for a data steward training workshop in Amsterdam in January 2020 to begin uploading pollen data to APD/Neotoma. The participants saw value in these combined efforts for assuring the longevity of a living, community-curated APD data resource.

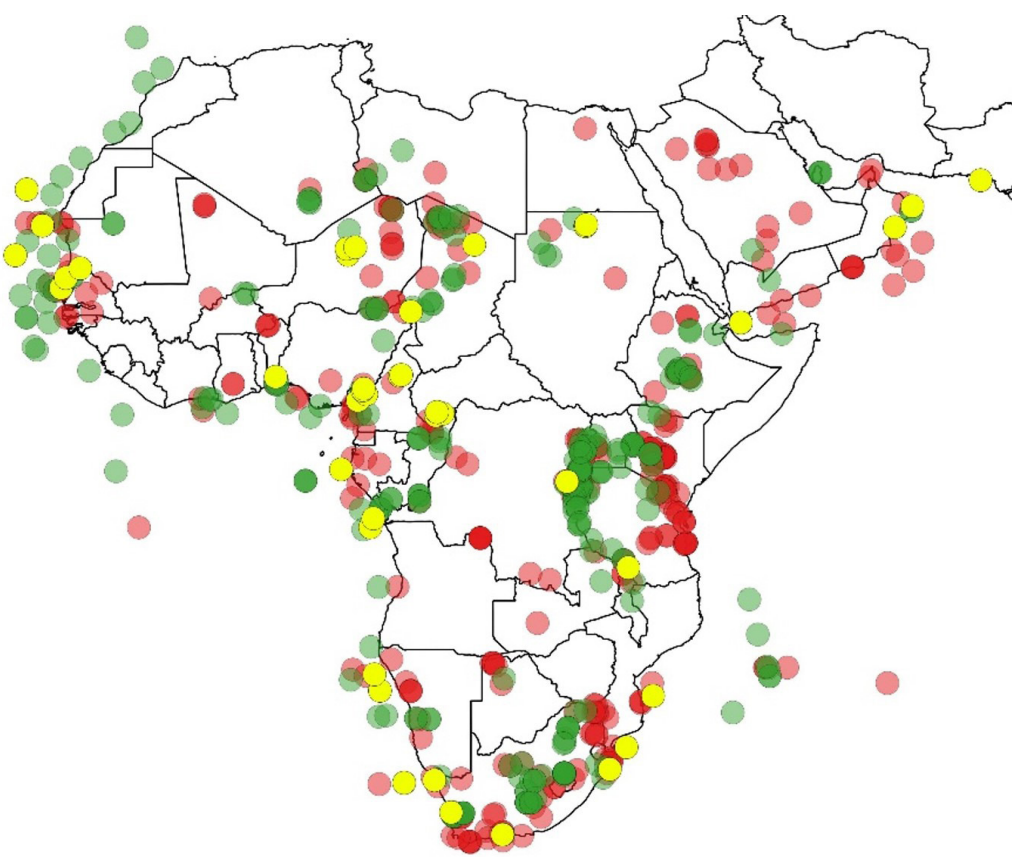

Figure 1: Paleoecological sites within the APD (green), datasets obtained but not yet within the APD (yellow), and known datasets not currently contributed to the APD (red dots). Northern Africa is in the European Pollen Database.

\section{Building a community}

Africa was divided into six sub-regions (Central, Southern, East, West, Arabia, Madagascar/Indian Ocean Islands, and marine), and regional experts identified records produced post-2007 and legacy datasets not included in the APD. The increase in research in Madagascar and Central Africa was notable, given prior underrepresentation of these regions. Specialists reported 365 known sites not in the APD, ranging in age from 1000 years to the Plio-Pleistocene. Modern samples (1985 entries) are also available, with more to be added. Some modern samples were collected as early as 1954 and should be used with caution to represent modern vegetation. In sum, these sites include a wealth of new information: marine and terrestrial, from diverse archives, many of which are well-dated with high temporal resolution. This will facilitate the burgeoning number of new studies and spatio-temporal meta-analysis of ecosystem-climate linkages, closing the gap with other regions.

Further, a governance body was launched, co-led by Sarah Ivory and Anne-Marie Lézine, with multiple scientists volunteering to serve as representatives and data stewards from each region. As the APD council is launched, an effort is being made to balance the numbers of African and non-African scientists as well as early-career scientists. Through representation within the APD and improved access to open data resources and software, we hope to build opportunities for young researchers from Africa.

\section{AFFILIATIONS}

Department of Geosciences, Pennsylvania State University, University Park, USA

'Laboratoire d'Océanographie et du Climat expérimentations et modélisations numériques (LOCEAN), CNRS, Paris, France 'Department of Earth and Environmental Sciences, University of Minnesota, Minneapolis, USA ${ }^{4}$ Department of Geography, University of Wisconsin, Madison, USA

CONTACT

Sarah Ivory: sji15@psu.edu

REFERENCES

Goring S et al. (2015) Open Quat 1: p.Art. 2 Myers N et al. (2000) Nature 403: 853-858 Niang l et al. (2014) In: Barros et al. (Eds) Climate change 2014: Impacts, Adaptation, and Vulnerability. Cambridge University Press, 1199-1265

Vincens A et al. (2007) Rev Palaeobot Palyn 145: 135-141 Wangai PW et al. (2016) Int J Sustain Built Environ 5 225-245

Wilkinson MD et al. (2016) Sci Data 3: 160018 Williams JW et al. (2018) PAGES Mag 26: 50-51 